\title{
Transient Stability Analysis of Grid-Tied Converters Considering PLL's Nonlinearity
}

\author{
Xiuqiang He, Hua Geng, and Shaokang Ma
}

\begin{abstract}
Transient instability events of grid-tied converters probably occur while riding through grid faults. During lowvoltage ride through (LVRT) period, seeing from the converter terminal towards the grid, the Thévenin equivalent grid impedance becomes pretty significant, accordingly making the converter terminal voltage highly sensitive to the output current. Under such circumstances, it is challenging for the converter to resynchronize with the grid via a phase-locked loop (PLL). This paper develops a reduced-order nonlinear model to elaborate on the dynamic synchronization characteristic of the converters. By considering the impact of grid impedance and analysing spatial vector tracking relation, resynchronization principle of the converters during LVRT is revealed. Besides, the impacts of circuit parameters and controller parameters, including residual grid voltage, grid impedance, current references, and PLL parameters, on the transient stability of the converters are investigated. The results are verified by simulation and experimental results.
\end{abstract}

Index Terms - Grid fault, low voltage ride through, phase-locked loop, power converter, synchronization, transient stability.

\section{INTRODUCTION}

G RID-TIED power converters are playing increasingly important roles in power systems, along with the rapid development of renewable power generation in recent years. Three-phase voltage source converters (VSCs), as one of the most crucial components of renewable energy generation units, often offer quite different performance from the conventional synchronous generator units, such as outstanding rapidity and controllability, but limited fault ride through capability [1], [2]. Grid codes for renewable power generation, in which various facets of the operating characteristics of grid-tied renewable energy generation units are standardized, have been formulated by independent system operators in order to ensure the power system security and stability [2]-[4]. One of the grid codes, namely low-voltage ride through (LVRT) requirement, requires renewable energy generation units to remain connected to the

Manuscript received January 25, 2019. This work was supported in part by the National Key R\&D Program of China under Grant 2016YFB0900300, and in part by the National Natural Science Foundation of China under Grants 61722307, U1510208, and 51711530235. This paper was presented in part at the 2018 IEEE International Power Electronics and Application Conference and Exposition (PEAC), Shenzhen, China, November 2018.

The authors are with the Department of Automation, Tsinghua University, Beijing, 100084, China (e-mail: he-xq16@mails.tsinghua.edu.cn; genghua@ tsinghua.edu.cn; msk15@mails.tsinghua.edu.cn).

Digital Object Identifier 10.24295/CPSSTPEA.2019.00005 grid and also output a specific reactive current to support the residual grid voltage during LVRT period [2]-[4].

When a grid fault occurs, the Thévenin equivalent grid impedance generally becomes considerable [5], resulting in weak connection between VSCs and the power grid [5]-[7]. Consequently, the VSC terminal voltage is easily affected by the VSC output current Conversely, the output current is also influenced by the terminal voltage since the current phase-angle is guided by the phaselocked loop (PLL) which is with the terminal voltage as the input. It has been reported that loss of synchronism (LOS) of the grid-tied VSCs probably happens during LVRT because of the dynamic interaction between the PLL and the terminal voltage [5]-[22]. Note that the LVRT requirements of the grid codes can no longer be satisfied once the LOS occurs.

During severe grid faults, i.e., under large disturbances, on one hand, initial states of the VSC systems will probably be random. On the other hand, state trajectories of the VSC systems often exhibit a large change magnitude. Therefore the nonlinearity in system components, especially PLL's nonlinearity, cannot be neglected in the stability assessment. In this regard, previous relevant small-signal stability studies of the VSCs [8]-[11] can not effectively address the transient stability issues.

The transient instability issues associated with the LOS have not drawn much attention. [12] and [13] made investigations on the presence of equilibrium points of the grid-tied VSCs during grid voltage sags, but dynamic synchronization characteristics were not taken into account. [14] and [15] also claimed that the transient stability of VSCs can be considered as the presence of post-fault equilibrium points. However, we found in [5]-[7] that there is an instability possibility for a grid-tied VSC due to improper initial states and poor dynamic performance in addition to the absence of equilibrium points.

To elaborate the instability issue in dynamic processes, [16]-[18] applied the concept of equal area criterion (EAC) to analyse the transient stability of VSCs during LVRT. Nevertheless, the results were conservative since the proportional unit of PLL was ignored in the EAC method. Lately, the research group of $\mathrm{X}$. Wang made innovative studies on the synchronization of grid-tied VSCs using the concept of phase portrait [19]-[22], which have created a new perspective for the transient stability studies of grid-tied VSCs. Besides, it should be noted that Lyapunov's direct method was also employed to assess the transient stability of grid-tied VSCs in [23]-[26]. Although transient stability can be judged through Lyapunov's direct method, the impacts of circuit parameters and controller parameters on the transient stability margin can only be investigated in a case-by-case way through the method, rather 
than evaluated systematically.

According to the literature review, although the dynamic synchronization process of VSCs has been investigated in [14]-[22], resynchronization principle of the VSCs during LVRT considering the impact of grid impedance on the PLL detecting ability has not been completely clarified. Furthermore, the effects of circuit parameters and controller parameters on the transient stability have not been extensively investigated. As an expanded version of our conference paper [27], this paper is devoted to filling this gap. Specifically, a reduced-order nonlinear model is derived and validated in Section II. A comprehensive stability analysis is performed in Section III, where the resynchronization principle is analysed through the spatial vector tracking relation from the PLL point of view. Also, the impacts of circuit parameters and controller parameters on the transient stability margin is quantitatively investigated. Sections IV and V show the simulation and experimental results, respectively, and Section VI concludes this paper.

\section{SySTEM ModeLing}

A grid-tied VSC system is shown in Fig. 1. PLL is utilized to detect the phase-angle and frequency of the VSC terminal voltage $\boldsymbol{u}_{a b c}$, and the estimated phase-angle $\theta_{p l l}$ is then used as the angle reference for the current control module. Fig. 1(b) depicts the dynamic interaction between the PLL and the VSC terminal voltage. The output of the PLL guides the phase-angle of the output current which makes the grid impedance produce a voltage drop $\Delta \boldsymbol{U}$. The grid impedance voltage drop $\Delta \boldsymbol{U}$ makes the terminal voltage $\boldsymbol{U}$ no longer stiff and highly sensitive to the output current once the grid impedance becomes considerable rather than ignorable.

When a grid fault occurs, a Thévenin equivalent circuit can be built from the fault point of view [5]. [5] indicated that the Thévenin equivalent grid impedance would become considerable if the grid fault is severe. In other words, severe faults would lead to weak connection between the VSC and the infinite-bus grid in the Thévenin equivalent circuit, even though the pre-fault grid is not weak [5].

Prior to modeling the VSC system for the transient stability analysis, several assumptions are made as follows [5]-[7], [12], [13], [16]-[22]:

1) The DC-link voltage of the VSC is considered to be constant, due to the fact that the configured chopper circuit [not shown in Fig. 1(a)] can maintain the DC-link voltage during LVRT.

2) The VSC with the current-controlled vector control can be regarded as a controlled current source, considering that the current loop bandwidth with appropriate current controller parameters is much higher than the PLL bandwidth.

3) Transmission line electromagnetic transient behaviors are neglected because of the fast dynamics.

4) The power grid is represented by an infinite bus with lumped impedance, and grid frequency dynamics are also neglected. Particular attention of this study is focused on the PLL dynamics.

A commonly used synchronous reference frame PLL (SRF-

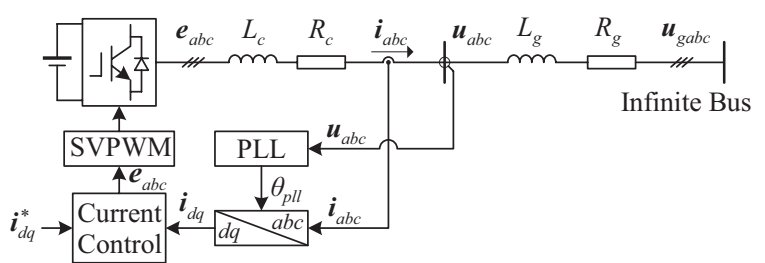

(a)

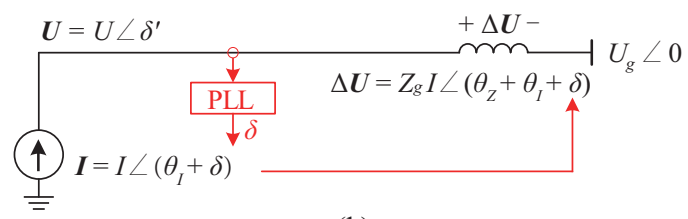

(b)

Fig. 1. Grid-tied VSC system. (a) PLL-based current control. (b) Illustration of the dynamic interaction between the PLL and the terminal voltage, where $\boldsymbol{U}$ and $\boldsymbol{I}$ are the phasors of $\boldsymbol{u}_{a b c}$ and $\boldsymbol{i}_{a b c}$

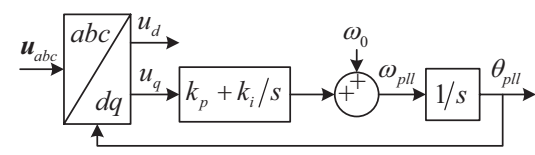

(a)

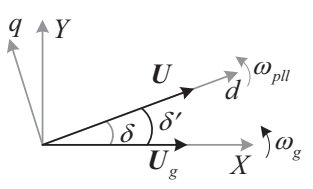

(b)

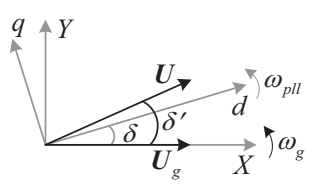

(c)

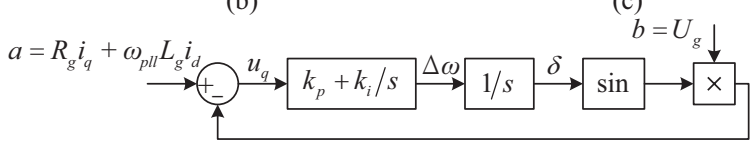

(d)

Fig. 2. (a) SRF-PLL diagram. (b) Steady-state relationship between the terminal voltage $\boldsymbol{U}$ and the two reference frames. (c) Dynamic relationship between the terminal voltage $\boldsymbol{U}$ and the two reference frames. (d) Reduced-order nonlinear system model.

PLL) is depicted in Fig. 2(a), where $\omega_{0}$ denotes the nominal frequency. Fig. 2(b) and (c) show the relationship between the PLL $d-q$ reference frame and the infinite-bus $X-Y$ synchronous reference frame. In the steady state, direction of the terminal voltage vector $\boldsymbol{U}$ coincides with PLL's $d$-axis, whereas they have different directions during dynamic processes. The included angle between the two reference frames in Fig. 2(b) [or (c)] is denoted $\delta$ and it can be accordingly obtained that

$$
d \delta / d t=\omega_{p l l}-\omega_{g} \triangleq \Delta \omega
$$

where $\omega_{\text {pll }}$ represents the PLL frequency and $\omega_{g}$ represents the grid frequency, and the difference between the two is defined as $\Delta \omega$. Furthermore, according to Fig. 2(a), PLL dynamics can be described as follows,

$$
d \Delta \omega / d t=d\left(\omega_{p l l}-\omega_{g}\right) / d t=d \omega_{p l l} / d t=k_{p} d u_{q} / d t+k_{i} u_{q}
$$

where $k_{p}$ and $k_{i}$ are the PLL parameters and $u_{q}$ is the $q$-axis 
voltage of the terminal voltage $\boldsymbol{u}_{a b c}$.

As shown in Fig. 2(b) or (c), transforming the grid voltage $\boldsymbol{U}_{g}$ from the $X-Y$ reference frame into the PLL $d-q$ reference frame gives rise to the $d$-axis and $q$-axis voltages of $\boldsymbol{U}_{g}$ as follows,

$$
\left[\begin{array}{l}
u_{g d} \\
u_{g q}
\end{array}\right]=\left[\begin{array}{cc}
\cos \delta & \sin \delta \\
-\sin \delta & \cos \delta
\end{array}\right]\left[\begin{array}{l}
U_{g} \\
0
\end{array}\right] .
$$

While neglecting the electromagnetic transient behaviors of the transmission lines, the mathematical relation between the VSC terminal voltage $\boldsymbol{u}_{d q}$ and output current $\boldsymbol{i}_{d q}$ can be expressed as follows,

$$
\left[\begin{array}{l}
u_{d} \\
u_{q}
\end{array}\right]=\left[\begin{array}{l}
u_{g d} \\
u_{g q}
\end{array}\right]+R_{g}\left[\begin{array}{l}
i_{d} \\
i_{q}
\end{array}\right]+\omega_{p l l} L_{g}\left[\begin{array}{c}
-i_{q} \\
i_{d}
\end{array}\right]
$$

where $\boldsymbol{u}_{d q}$ and $\boldsymbol{i}_{d q}$ are the counterparts of $\boldsymbol{u}_{a b c}$ and $\boldsymbol{i}_{a b c}$ transformed into the PLL $d-q$ reference frame, respectively. Besides, $R_{g}$ and $L_{g}$ represent the resistive and inductive components of the lumped grid impedance. It is noted that the grid impedance is from the Thévenin equivalent circuit, therefore it would change with a transmission line ground fault [5], [28].

From (3) and (4), it can be further derived that

$$
u_{q}=R_{g} i_{q}+\omega_{p l l} L_{g} i_{d}-U_{g} \sin \delta \triangleq a-b \sin \delta
$$

From (5), it can be found that $a$ is an offset term in $u_{q}$, which is introduced by the grid impedance voltage drop. Detailed expressions of $a$ and $b$ are as follows,

$$
\left\{\begin{array}{l}
a=R_{g} i_{q}+\omega_{p l l} L_{g} i_{d}=Z_{g} I \sin \left(\theta_{Z}+\theta_{I}\right) \\
b=U_{g}
\end{array}\right.
$$

where $Z_{g}$ denotes the grid impedance magnitude; $I$ denotes the current amplitude; $\theta_{I}$ and $\theta_{Z}$ (impedance angle) are expressed as follows,

$$
\begin{gathered}
I=I \angle\left(\theta_{I}+\delta\right), \theta_{I}=\arctan \left(i_{q} / i_{d}\right) \\
Z_{g}=Z_{g} \angle \theta_{Z}, \theta_{Z}=\arctan \left(\omega_{p l l} L_{g} / R_{g}\right) .
\end{gathered}
$$

The differential equations (1) and (2) combining the algebraic equation (5) constitute the reduced-order nonlinear system model, as shown in Fig. 2(d). In addition, value of the currents $i_{d}$ and $i_{q}$ in the model should be stated, in order to analyse the transient stability based on the model. Since the VSC system is regarded as a controlled current source, it can be considered that the output current $\boldsymbol{i}_{d q}$ is directly specified by the current references. Taking into account the unity power factor control, the pre-fault current references can be denoted as

$$
\begin{aligned}
& i_{d_{\_ \text {normal }}}=i_{d_{0}} \\
& i_{q_{\text {_normal }}}=0
\end{aligned}
$$

where $i_{d 0}$ denotes the pre-fault steady-state active current, and
TABLE I

VSC Model Parameters

\begin{tabular}{cccc}
\hline \hline Parameter & Per-unit value & Parameter & Per-unit value \\
\hline$L_{g}$ & 0.08 & Residual grid voltage & 0.05 or 0.10 \\
$R_{g}$ & 0.30 & $k_{p}$ (PLL) & 100 \\
$L_{c}$ & 0.15 & $k_{\text {(PLL) }}$ & 1000 \\
$R_{c}$ & 0.005 & $k_{p}$ (current controller) & 2 \\
$i_{d_{\text {nnormal }}}$ & 1.0 & $k_{i}$ (current controller) & 10 \\
$i_{q_{-} \text {normal }}$ & 0.0 & Simulation step size & $5 \times 10^{-6} \mathrm{~s}$ \\
$i_{d_{-} \text {fault }}$ & 0.0 & $i_{q_{-} \text {fault }}$ & -1.0 \\
\hline \hline
\end{tabular}
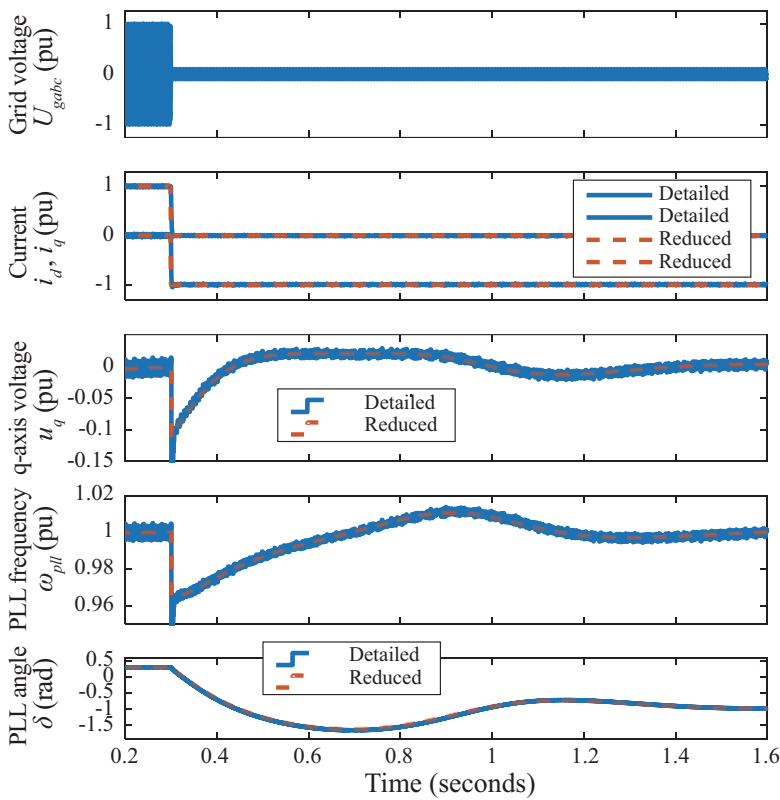

Fig. 3. Validation of the reduced-order model by comparison with the detailed model, where the grid voltage dips to 0.10 p.u.

the VSC outputs zero reactive current under normal operating conditions. The output current during LVRT conditions is directly determined by the grid codes. For example, $i_{q}{ }^{*}$ is set to -1.0 p.u. if the PCC (point of common coupling) voltage dips larger than $50 \%[3]$ and $i_{d}{ }^{*}$ is often set to zero considering the output current capability, i.e.,

$$
\begin{gathered}
i_{d_{-} \text {fault }}=0 \\
i_{q_{-} \text {fault }}=-1 \text { p.u. }
\end{gathered}
$$

To demonstrate the adequacy of the developed reduced-order nonlinear model, the model is validated by comparing its timedomain responses with those of the detailed electromagnetic transient (EMT) model. Parameters of the detailed model are summarized in Table I.

Figs. 3 and 4 give the simulation results. In Fig. 3, the grid voltage dips from the nominal condition to 0.10 p.u. at $0.3 \mathrm{~s}$, and the VSC output current quickly changes in the meantime according to the reference. Specifically, the reactive current instantaneously changes from 0 to -1 p.u., and the active current changes from $i_{d 0}$ to 0 . Fig. 3 shows that the PLL is able to quickly resynchronize with the grid after the grid voltage sag. 

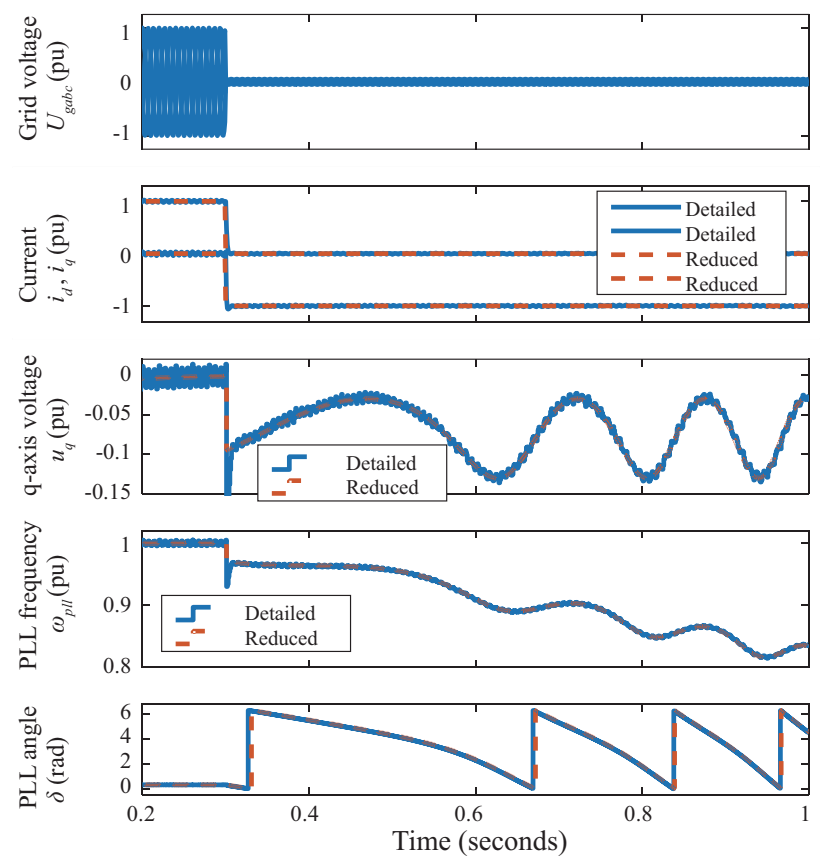

Fig. 4. Validation of the reduced-order model by comparison with the detailed model, where the grid voltage dips to 0.05 p.u., making the VSC system become unstable.

In Fig. 4, a more severe grid voltage sag than that in Fig. 3 occurs, i.e., only 0.05 p.u. residual grid voltage. Simulation results in Fig. 4 indicate that the PLL is not able to resynchronize with the grid after the grid voltage sag. Hence, the VSC system becomes unstable.

From Figs. 3 and 4, it can also be observed that the timedomain responses of the reduced-order model and those of the detailed EMT model are highly consistent, therefore verifying the adequacy of the reduced-order model. Based on the reduced-order model, the transient stability analysis can be easily performed.

\section{Transient Stability Analysis}

The reduced-order model is comprised of (1), (2), and (5). (1) and (2) describe the PLL dynamic nonlinear characteristic, whereas the zero-crossing point of (5), i.e., $u_{q}=0$, determines the steady-state equilibrium point of the system. The transient stability of the system will be analysed from two points of views, which are the equilibrium point analysis and the dynamic characteristic analysis.

\section{A. Steady-State Equilibrium Point Analysis}

According to (5), the steady-state equilibrium point of the VSC system satisfies the condition that

$$
u_{q}=a-b \sin \delta=0 .
$$

Several examples of the equilibrium point are depicted in Fig. 5, where there are two types of zero-crossing points. The small-signal stability analysis is performed to distinguish between the unstable equilibrium points (UEPs) and the stable

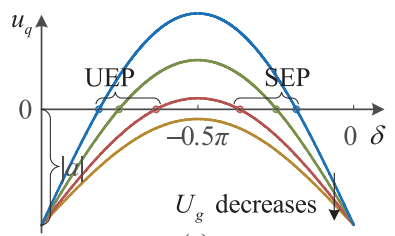

(a)

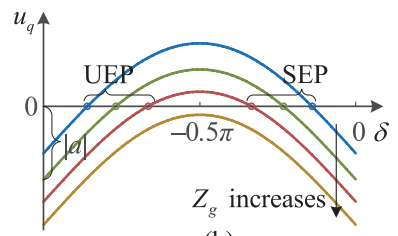

(b)
Fig. 5. Illustration of equilibrium points. (a) Grid voltage $U_{g}$ decreases. (b) Grid impedance magnitude $Z_{g}$ increases.

equilibrium points (SEPs). The small-signal state variables $\tilde{\delta}$ and $\Delta \tilde{\omega}$ can be denoted as follows,

$$
\left\{\begin{array}{l}
\delta=\delta_{0}+\tilde{\delta} \\
\Delta \omega=0+\Delta \tilde{\omega} .
\end{array}\right.
$$

Based on the nonlinear model consisting of (1), (2), and (5), and the equilibrium point in (12), the small-signal model can be derived, as shown in (13).

$\left[\begin{array}{c}\dot{\tilde{\delta}} \\ \dot{\tilde{\omega}}\end{array}\right]=\left[\begin{array}{cc}0 & 1 \\ -\frac{k_{i} U_{g} \cos \delta_{0}}{1-k_{p} L_{g} i_{d}} & -\frac{k_{p} U_{g} \cos \delta_{0}-k_{i} L_{g} i_{d}}{1-k_{p} L_{g} i_{d}}\end{array}\right]\left[\begin{array}{c}\tilde{\delta} \\ \tilde{\omega}\end{array}\right]$

Considering that $1-k_{p} L_{g} i_{d}>0$ almost always holds true, to meet the small-signal stability criterion, the following conditions should be satisfied,

$$
\begin{gathered}
\cos \delta_{0}>0 \\
k_{p} U_{g} \cos \delta_{0}>k_{i} L_{g} i_{d} .
\end{gathered}
$$

(15) can further give rise to

$$
\cos \delta_{0}>k_{i} L_{g} i_{d} /\left(k_{p} U_{g}\right) \approx 0
$$

which is approximately equivalent to (14). Thus, (14) indicates the zone where SEPs are located, as shown in Fig. 5.

From Fig. 5(a), it can be observed that the curve amplitude $b$ decreases along with the decrease of the grid voltage $U_{\mathrm{g}}$ (because $b=U_{g}$ ). In Fig. 5(b), the absolute value of the offset term $|a|$ increases along with the increase of the grid impedance magnitude $Z_{g}$.

If $|a|$ is larger than $b$, caused by a quite severe grid sag or a considerable post-fault grid impedance, there will be no equilibrium point. Then, it will be impossible to maintain the stability of the system whether PLL [29] or frequency-locked loop (FLL) [30], [31] is adopted. The critical equilibrium point corresponds to $|a|=b$.

From Fig. 5, it can also be found that the smaller the absolute offset term $|a|$, and the larger the residual grid voltage amplitude $b$, the higher the stability margin. Besides, (6) indicates that four variables associated with the output current and the grid impedance have impacts on the offset term. Specifically, both a large current amplitude $I$ and a large grid impedance magnitude 


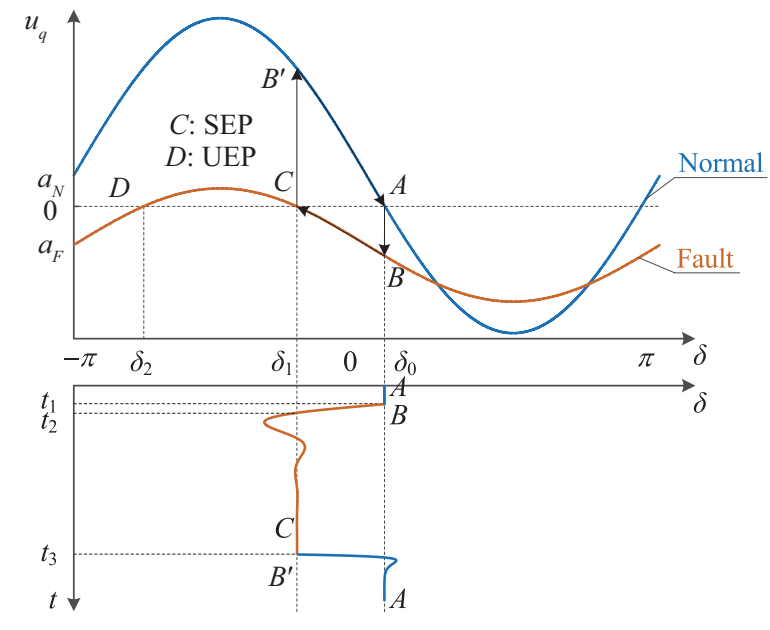

Fig. 6. Illustration of the dynamic synchronization processes of a VSC subjected to a grid voltage sag at $t_{1}$ and grid voltage recovery at $t_{3}$. Note that $a_{N}$ and $a_{F}$ denote the offset terms corresponding to the normal and fault operating conditions, respectively.

$Z_{g}$ are able to facilitate a large offset term, which accordingly shrinks the stability margin.

When it comes to the relation between the offset term and $\theta_{I}$ or $\theta_{Z}$, (6) manifests that the relation is not linear. In particular, the offset term equals zero only if $\theta_{I}+\theta_{Z}=0$. In general, $\theta_{I}=-0.5 \pi$ when $i_{d}=0$ is set during LVRT. In this regard, the offset term equals zero only if $\theta_{Z}=0.5 \pi$. Nonetheless, $\theta_{Z}$ is random in actual grid fault scenarios, which is determined by actual fault points and short-circuit resistance.

\section{B. Resynchronization Principle Analysis}

Without taking into account the impact of the grid impedance on the PLL, grid synchronization principle as well as dynamic performance of PLLs have been extensively investigated in previous studies [29]. However, it is not enough to guarantee the transient stability of the VSC systems, especially for ones connected to the high-impedance weak grid. Here, from the perspective of spatial vector tracking relation, a resynchronization principle analysis of the VSCs during LVRT is conducted to clarify the stability principle associated with PLL's nonlinearity by considering the impact of the grid impedance.

In Fig. 2(d), the grid impedance introduces the offset term $a$ into the input of the PLL. There is no doubt that the offset term makes the PLL no longer ideal since the input signal $u_{q}$ is no longer a standard unbiased sinusoidal function related to $\delta$. Therefore, it has effects on the synchronization performance of the PLL.

Fig. 6 illustrates an example of the dynamic synchronization process of a VSC subjected to a grid voltage sag and also a grid voltage recovery. When the grid voltage sag occurs at $t_{1}$, the operating curve switches from the blue curve (Normal condition) to the orange curve (Fault condition), and the operating point steps from $A$ to $B$. There are two steady-state equilibrium points, i.e., $C$ and $D$, after the grid voltage sag. Note that point $C$ is stable whereas $D$ is unstable. Then, the

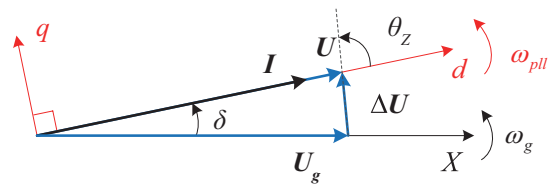

(a)

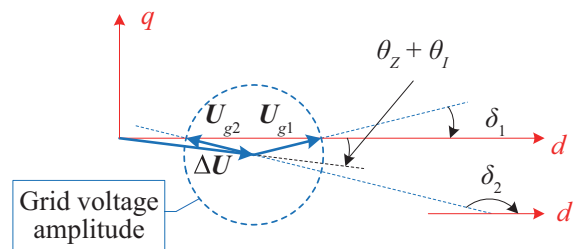

(b)
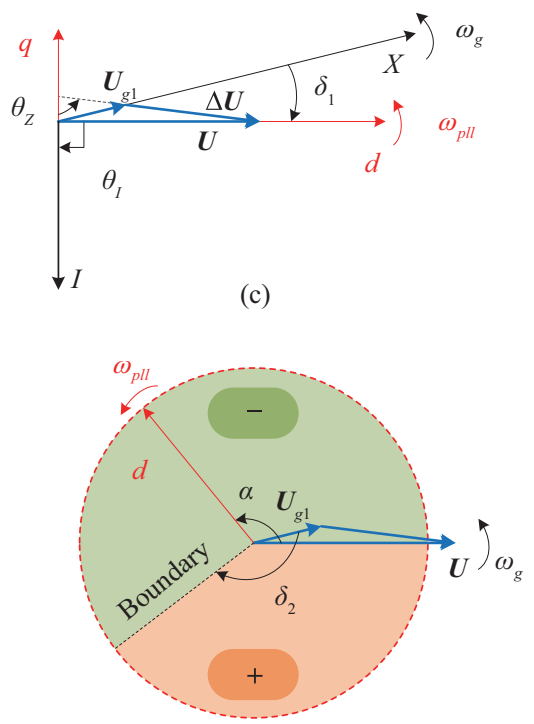

(d)

Fig. 7. (a) Spatial vector relation in the pre-fault steady state. (b) There are two steady-state equilibrium points after the grid voltage sag, i.e., $\boldsymbol{U}_{g 1}$ (stable) and $\boldsymbol{U}_{\mathrm{g} 2}$ (unstable), where $\delta_{1}+\delta_{2}=-\pi$. (c) Spatial vector relation corresponding to the post-fault stable steady-state equilibrium point. (d) Dynamic synchronization process illustration.

operating point moves towards $C$ because $B$ is not a steady-state point, accompanied by a decelerating process of the PLL. The operating point crosses $C$ at $t_{2}$, thereafter the PLL frequency begins to increase due to $u_{q}$ becomes positive. Finally, the operating point reaches the equilibrium point $C$ due to the damping effects. It is supposed that $\delta$ would not exceed the UEP $D$ in the accelerating process, otherwise the operating point will fall into the adjacent inversely regulating areas. When the grid fault is cleared at $t_{3}$, similar dynamic synchronization process is performed.

Considering the impact of the grid impedance, resynchronization principle of the VSC is elaborated using spatial vector tracking relation. Fig. 7(a) shows the pre-fault steadystate spatial vector relation, where $\omega_{g}=\omega_{\text {pll }}$. Fig. 7(b) shows that there are two steady-state equilibrium points after the grid voltage sag. The grid impedance voltage drop $\Delta \boldsymbol{U}$ is uniquely identified with respect to the $d-q$ reference frame, provided that both grid voltage impedance $\boldsymbol{Z}_{g}$ and VSC output current 
$I$ are determined. The dashed circle denotes the grid voltage amplitude $U_{g}$, resulting in two intersections with the $d$-axis, hence corresponding to two grid voltage vectors, $\boldsymbol{U}_{g 1}$ and $\boldsymbol{U}_{g 2}$. It has been known that only the equilibrium point corresponding to $\boldsymbol{U}_{g 1}$ is stable.

Fig. 7(c) illustrates the post-fault steady-state vector relation, where $\omega_{g}=\omega_{\text {pll }}$. As shown in Fig. 7(d), the goal of the PLL is to track the determined voltage vector $\boldsymbol{U}$, i.e., the postfault steady-state terminal voltage vector, during the dynamic synchronization process. If the $d$-axis of the PLL reference frame surpasses the target vector $\boldsymbol{U}$ and the lead angle $\alpha$ is within the boundary, the PLL will decelerate. On the flip side, if the $d$-axis lags behind the target vector $\boldsymbol{U}$ and the lag angle is within the boundary, the PLL will accelerate.

It should be noted that the accelerating and decelerating areas are mismatched because of the offset term $a$ in the PLL input signal $u_{q}$. Owing to the mismatched accelerating and decelerating areas, the lead angle $\alpha$ might become so large that it traverses the boundary and then falls into the adjacent inversely regulating areas. As a result, an additional round of tracking path is caused, which, unfortunately, leads to the accelerating and decelerating areas being further mismatched. Therefore, the tracking capability of the PLL is further deteriorated, and loss of synchronism (LOS) potentially occurs.

To sum up, the coordinate transformation (3) incorporates sinusoidal functions, hence introducing both periodicity and nonlinearity. The periodicity leads to the accelerating and decelerating areas being adjacent to each other. The nonlinearity causes the PLL dynamic performance inconsistency at different operating points. Given that the developed nonlinear model is second-order, it is feasible to elaborate the dynamic performance by using the concept of inertia and damping.

\section{Damping Characteristic Analysis}

Transform the developed nonlinear model into a typical form of motion equation as follows,

$$
\begin{gathered}
J_{e q} d \omega_{p l l} / d t=u_{q}-D_{e q}\left(\omega_{p l l}-\omega_{g}\right) \\
d \delta / d t=\Delta \omega
\end{gathered}
$$

where the equivalent inertia and damping coefficients are

$$
\begin{gathered}
J_{e q}=\left(1-k_{p} L_{g} i_{d}\right) / k_{i} \\
D_{e q}=k_{p} U_{g} \cos \delta / k_{i}, k_{i} \neq 0 .
\end{gathered}
$$

Actually, since $k_{p} L_{g} i_{d}<<1,(18)$ can be further simplified as

$$
\begin{gathered}
J_{e q} \approx 1 / k_{i} \\
D_{e q}=k_{p} U_{g} \cos \delta / k_{i}, k_{i} \neq 0 .
\end{gathered}
$$

As shown in Fig. 6, the damping term plays an important role in weakening and gradually suppressing the oscillation during the dynamic synchronization process. If $k_{p}$ equals zero, then $D_{e q}$ will become zero, and it can be accordingly believed that the damping effect actually originates from the proportional unit of

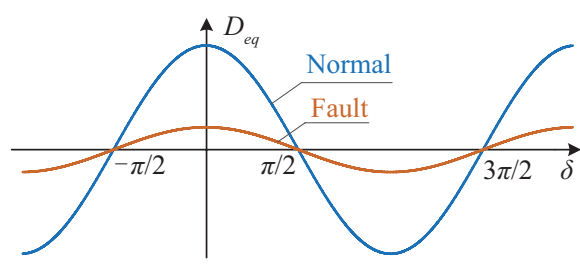

Fig. 8. Change of $D_{e q}$ with $\delta$, where $D_{e q}>0$ corresponds to positive damping zones whereas $D_{e q}<0$ corresponds to negative zones.

the PLL. It should be noted that [16]-[18] employed the concept of equal area criterion to analyse the stability of grid-tied VSCs based on (17). Since the damping term was neglected, the results were relatively conservative.

According to (19), it can be found that a small $k_{p}$, a large $k_{i}$, and a small $U_{g}$ are able to make the damping effect weak, therefore deteriorate the stability. Especially, the sign of the damping coefficient is directly decided by the PLL angle $\delta$. Fig. 8 shows the relation between $D_{e q}$ and $\delta$. Once the operating point enters negative damping zones, loss of synchronism (LOS) is very likely to occur. In addition, (19) seemly reveals that the grid impedance has no effect on the damping coefficient. However, it should be noted that grid impedance is able to produce impacts on the PLL angle $\delta$, accordingly have impacts on the damping characteristics.

\section{Inertia Characteristic Analysis}

From (19), it can be found that the equivalent inertia coefficient $J_{e q}$ is directly related to the integral coefficient $k_{i}$ of the PLL. Specifically, $J_{e q}$ is inversely proportional to $k_{i}$. Therefore, a large $k_{i}$ is able to lead to a small $J_{e q}$, which, as a result, might cause potential oscillations with large amplitude. In other words, a large $k_{i}$ is harmful to the stability of grid-tied VSC during LVRT.

\section{Simulation Results}

Simulations are conducted to verify the above results regarding the impacts of circuit parameters and controller parameters on the transient stability of VSCs. Simulations are performed based on a VSC switching-cycle average model with parameters given in Table I. On this basis, bandwidth of the PLL and the current loop can be calculated.

\section{A. Different Grid Voltage Sags}

Simulating three degrees of grid voltage sags, i.e., $85 \%, 90 \%$, and $95 \%$, the simulation results are shown in Fig. 9. It can be found that the VSC cannot resynchronize with the grid when the $95 \%$ grid voltage sag occurs. Actually, it is because that there is no equilibrium point after the grid voltage sag.

Besides, when the grid voltage dips $85 \%$ and $90 \%$, it can be confirmed that there are indeed equilibrium points, and the system are stable in these two cases. It can also be found that a large residual grid voltage can not only help the present of postfault equilibrium points, but also facilitate the damping effect for a fast convergence. 

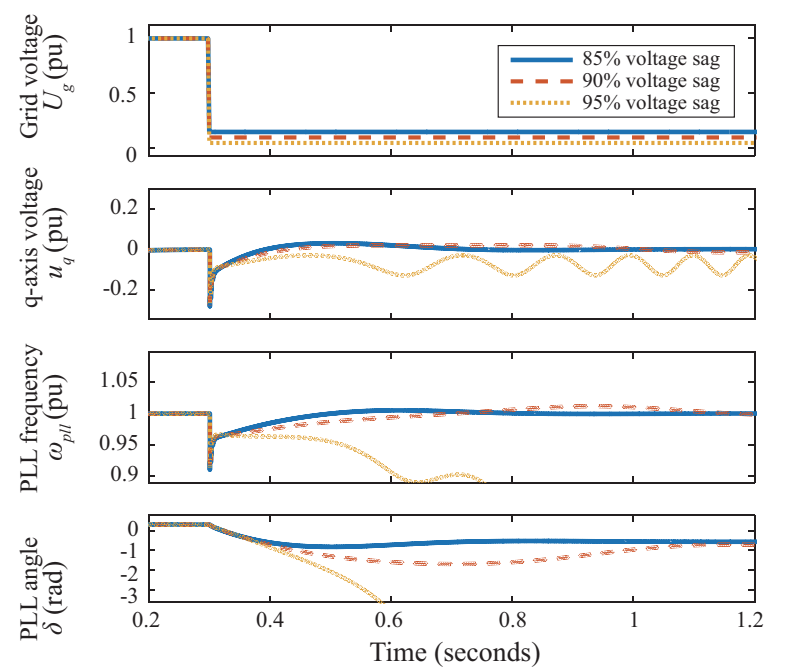

Fig. 9. Simulation results of $85 \%, 90 \%$, and $95 \%$ grid voltage sags.
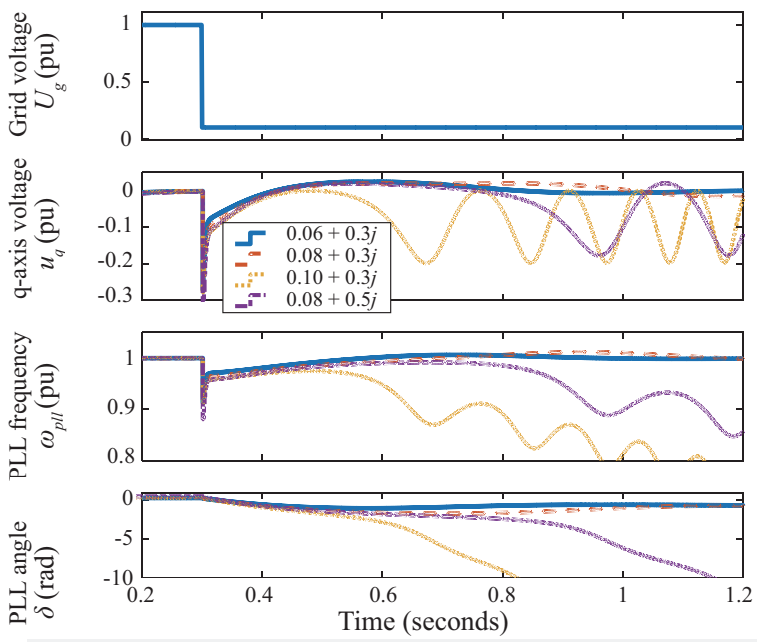

Fig. 10. Simulation results with different post-fault grid impedances, including $\boldsymbol{Z}_{g}=0.06+0.30 j, 0.08+0.30 j, 0.10+0.30 j$, and $0.08+0.50 j$.

To sum up, a large residual grid voltage after grid faults contributes to the enhancement of the transient stability.

\section{B. Different Grid Impedance}

Simulating four groups of grid impedance, i.e., $\boldsymbol{Z}_{g}=0.06+0.30 j$, $0.08+0.30 j, 0.10+0.30 j$, and $0.08+0.50 j$ after the same $90 \%$ grid voltage sag with the same current reference $i_{d_{-} \text {fault }}+j i_{q_{-} \text {fault }}=0.0-1.0 j$, the simulation results are shown in Fig. 10. With the increase of the resistive component, it can be observed that the equilibrium point probably becomes absent, thus the system tends to become unstable after the fault.

Besides, Fig. 10 shows that the increase of the inductive component is also able to deteriorate the stability. Although the inductive component of the grid impedance has no effect on the existence of equilibrium points, it is able to affect the initial system state. Specifically, a larger inductive component of the grid impedance makes the initial system state move away
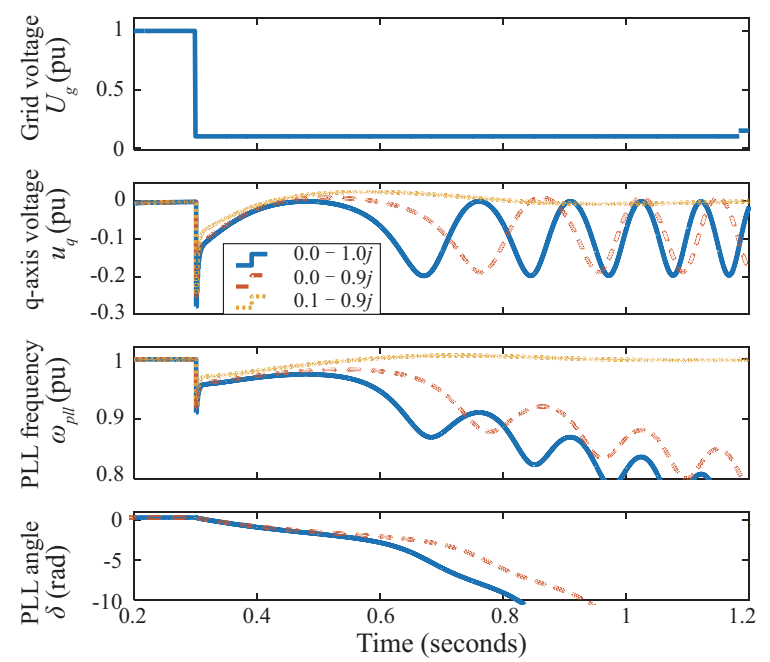

Fig. 11. Simulation results with different current references, including $0.0-1.0 j$, $0.0-0.9 j$, and $0.10-0.9 j$.

from the post-fault equilibrium point, hence leading to a larger accelerating area, as shown in Fig. 6.

To sum up, the transient stability margin is reduced with the increase of grid impedance, either in the resistive or the inductive component. In other words, the weak connection between VSCs and the power grid is indeed harmful to the transient stability of VSCs.

\section{Different Current References}

Simulating three groups of current reference, i.e., $i_{d_{\text {fault }}}+j i_{q_{\text {fault }}}=$ $0.0-1.0 j, 0.0-0.9 j$, and $0.10-0.9 j$ after the same $90 \%$ grid voltage sag with the same post-fault grid impedance $0.1+0.3 j$, the simulation results are shown in Fig. 11. For the first group of current reference, there exists a critical equilibrium point. The system becomes unstable because of overshoot. For the second group, although the stable equilibrium point is recreated by reducing the reactive current and thereby reducing the offset term in the $q$-axis voltage $u_{q}$, the system still becomes unstable due to the small stability margin. Furthermore, an additional active current is generated in the third group, which further reduces the offset term in $u_{q}$, and the system accordingly becomes stable.

To sum up, a small reactive current as well as a nonzero active current is conducive to improving the stability margin. It is because they can help to decrease the offset term in the $q$-axis voltage.

\section{Different PLL Parameters}

Figs. 12 and 13 show the impacts of PLL parameters on the transient stability. In Fig. 12, the system with $k_{p}=80$ becomes unstable after the grid voltage sag, since a small proportional coefficient gives rise to a small damping coefficient [see (19)], therefore a poor damping effect. The stability is improved with the increase of the PLL proportional coefficient. 

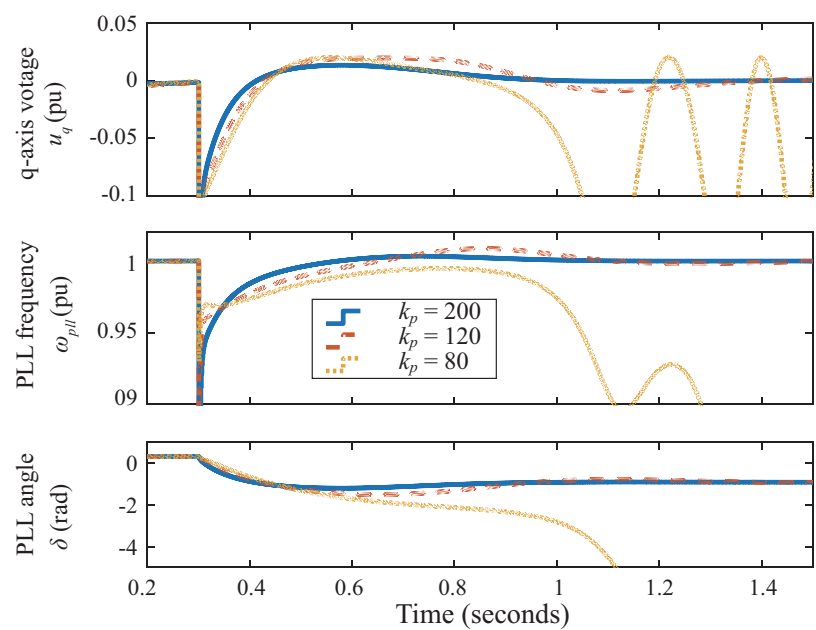

Fig. 12. Simulation results with different PLL proportional coefficients, including $k_{p}=200,120$, and 80 , but the same integral coefficient $k_{i}=1000$.

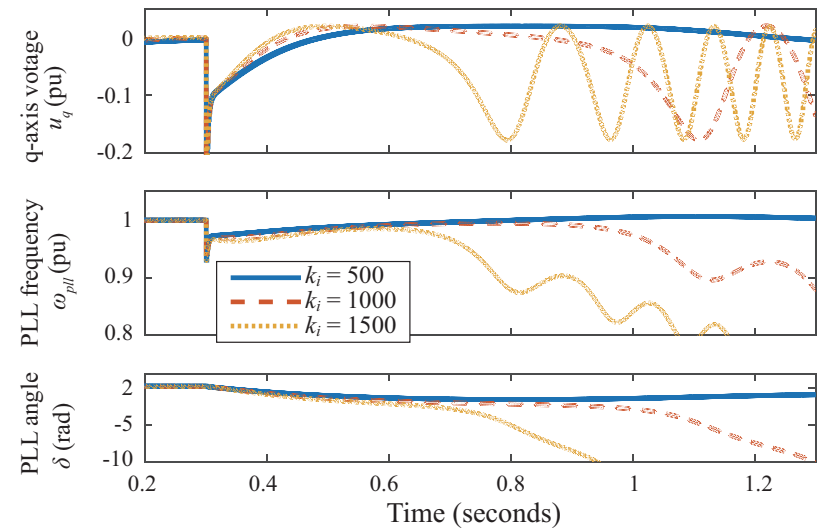

Fig. 13. Simulation results with different PLL integral proportional coefficients, including $k_{i}=500,1000$, and 1500, but the same proportional coefficient $k_{p}=80$.

Fig. 13 shows that the stability is enhanced with the decrease of the PLL integral coefficient. Actually, it is because that a small integral coefficient yields a large damping coefficient. To sum up, the above results completely coincide with the findings from (19), i.e., both a large $k_{p}$ and a small $k_{i}$ during grid voltage sags, are able to make the damping effect strong and therefore improve the transient stability.

It should be noted that the above results are only valid under the condition of ignorable current control dynamics. Once the PLL bandwidth overlaps with the current control bandwidth, both PLL dynamics and current control dynamics should be taken into account while analysing the transient stability.

\section{EXPERIMENTAL RESULTS}

The stability analysis has also been verified in an actual three-phase $1.0 \mathrm{~kW}$ PWM converter system with a DSP digital control platform. The setup of the experimental system is shown in Fig. 14 with parameters given in Table II. Note that both the resistive and inductive components of the grid impedance are large enough to mimic quite weak connection between the VSC

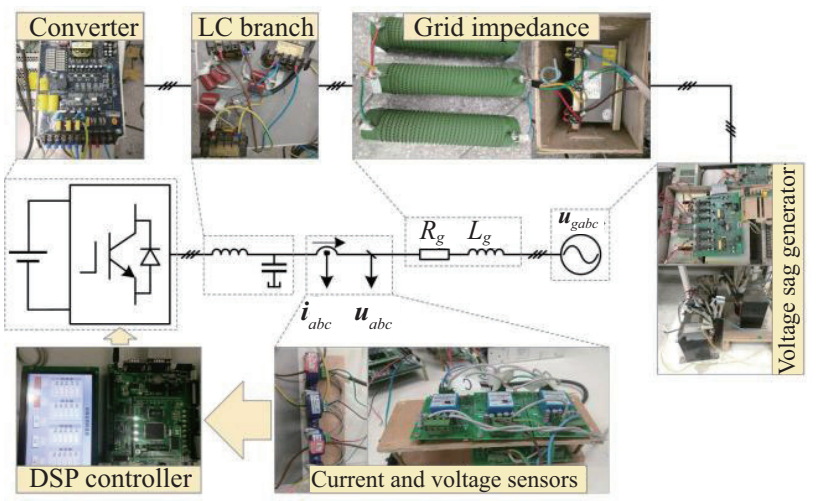

Fig. 14. Experimental setup.

TABLE II

EXPERIMENTAL System PARAMETers

\begin{tabular}{lcc}
\hline \hline Capability & $1 \mathrm{~kW}$ & \\
Nominal line-to-line voltage & $170 \mathrm{~V}$ & \\
Grid resistance & $3.5 \Omega$ & 0.121 p.u. \\
Grid inductance & $20 \mathrm{mH}$ & 0.217 p.u. \\
\hline \hline
\end{tabular}

and the grid.

From the viewpoint of enhancing the transient stability of grid-tied VSCs, regulating the current references and PLL parameters is a very easy way. For this purpose, two experimental cases are set to further verify: 1) the impact of current references and 2) the impact of PLL parameters on the transient stability of grid-tied VSCs.

\section{A. Impact of Current References}

The main attention of this case is focused on the active current reference, considering that there is almost no strict requirement on the active current output in most of the current LVRT codes. In Fig. 15(a), (b), and (c), the active current references are $0.0,1.0$, and $1.6 \mathrm{~A}$, respectively, with the same reactive current reference of -5.1 A during the grid voltage sag from $170 \mathrm{~V}$ to $14.2 \mathrm{~V}$. As shown in Fig. 15(a) or (b), the system is unstable with the active current of $0.0 \mathrm{~A}$ or $1.0 \mathrm{~A}$, because there is no equilibrium point. Note that $k_{i}$ is set to zero here, therefore the PLL frequencies undergo continuous oscillations instead of gradual deviations. In Fig. 15(c), the system is stable when the equilibrium point is recreated by a larger active current than that in Fig. 15(a) and (b).

\section{B. Impact of PLL Parameters}

The main attention of this case is focused on the PLL integral coefficient as an example. In Fig. 16(a), (b), and (c), the PLL integral coefficients are 2000,1500, and 1000, respectively, with the same PLL proportional coefficient of 25 during the grid voltage sag. From Fig. 16, it is noticed that the stability is enhanced with the decrease of the PLL integral coefficient, due to the increase of the damping effect. The experimental results accordingly further verify the stability analysis. 


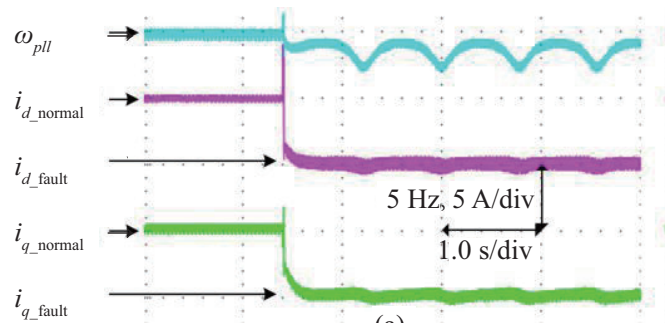

(a)

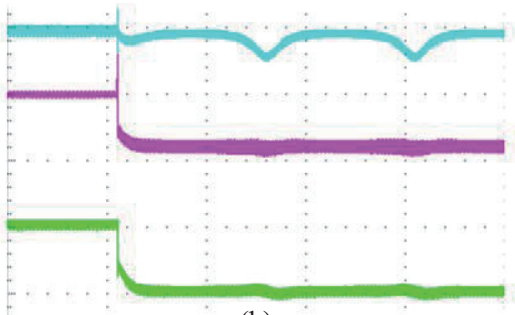

(b)

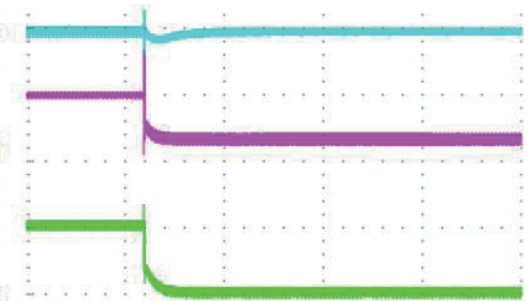

(c)

Fig. 15. Experimental results with different active current references during a grid fault. (a) $i_{d_{-} \text {fault }}=0.0 \mathrm{~A}$. (b) $i_{d_{-} \text {fault }}=1.0 \mathrm{~A}$. (c) $i_{d_{-} \text {fault }}=1.6 \mathrm{~A}$.

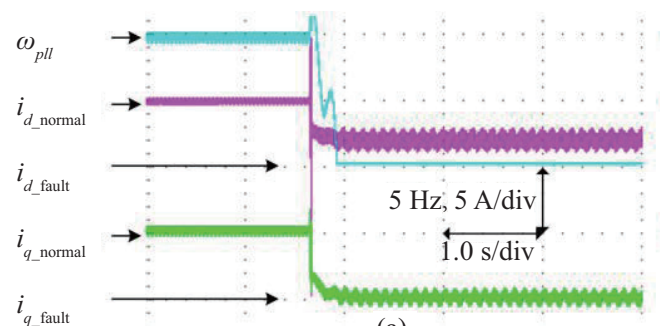

(a)

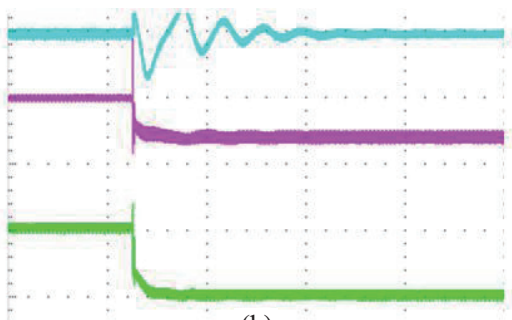

(b)

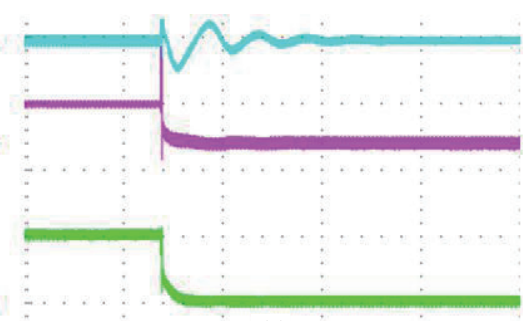

(c)

Fig. 16. Experimental results with different PLL integral coefficients during a grid fault. (a) $k_{i}=2000$. (b) $k_{i}=1500$. (c) $k_{i}=1000$.

\section{Conclusions}

Loss of synchronism of the grid-tied VSCs probably occurs during severe grid voltage sags. This paper develops a reducedorder nonlinear model to describe the dynamic synchronization process of VSCs. Based on the model, it is clarified that the post-fault grid impedance voltage drop has a significant effect on the transient stability, since it introduces an offset term in the $q$-axis voltage (i.e., PLL input signal). By determining the target terminal voltage vector, the resynchronization principle is analysed from the PLL point of view, according to spatial vector tracking relation. Moreover, from the perspective of equilibrium points and dynamic characteristics, the impacts of grid parameters, current references, and PLL parameters on the transient stability are analysed and verified. The results of this study could be conducive to guiding the designs of current references and PLL parameters for efficiently addressing grid faults.

\section{REFERENCES}

[1] G. Qi, "Improved control strategy of interlinking converters with synchronous generator characteristic in islanded hybrid AC/DC microgrid," CPSS Trans. Power Electron. Appl., vol. 2, no. 2, pp. 149-158, Jun. 2017.

[2] H. Geng, C. Liu, and G. Yang, "LVRT capability of DFIG-based WECS under asymmetrical grid fault condition," IEEE Trans. Ind. Electron., vol. 60, no. 6, pp. 2495-2509, 2013.

[3] Grid code-high and extra high voltage, E. ON Netz GmbH, 2006. Tech. Rep., [EB/OL].

[4] M. N. I. Sarkar, L. G. Meegahapola, and M. Datta, "Reactive power management in renewable rich power grids: A review of gridcodes, renewable generators, support devices, control strategies and optimization algorithms," IEEE Access, vol. 6, pp. 41458-41489, 2018.

[5] S. Ma, H. Geng, L. Liu, G. Yang, and B. C. Pal, "Grid-synchronization stability improvement of large scale wind farm during severe grid fault," IEEE Trans. Power Syst., vol. 33, no. 1, pp. 216-226, 2018.
[6] H. Geng, L. Liu, and R. Li, "Synchronization and reactive current support of PMSG based wind farm during severe grid fault," IEEE Trans. Sustain. Energy, vol. 9, no. 4, pp. 1596-1604, Oct. 2018.

[7] S. Ma, H. Geng, G. Yang, and B. Liu, "Experimental validation of adaptive current injecting method for grid-synchronization improvement of grid-tied REGS during short-circuit fault," in Proc. IPEC-Niigata, 2018.

[8] J. Hu, B. Wang, W. Wang, H. Tang, Y. Chi, and Q. Hu, "Small signal dynamics of DFIG-based wind turbines during riding through symmetrical faults in weak AC grid," IEEE Trans. Energy Convers., vol. 32, no. 2, pp. 720-730, 2017.

[9] B. Wen, D. Dong, D. Boroyevich, R. Burgos, P. Mattavelli, and Z. Shen, "Impedance-based analysis of grid-synchronization stability for three-phase paralleled converters," IEEE Trans. Power Electron., vol. 31, no. 1, pp. 26-38, 2016.

[10] M. Davari, Y. A.-R. I. Mohamed, "Robust vector control of a very weak grid-connected voltage-source converter considering the phaselocked loop dynamics," IEEE Trans. Power Electron., vol. 32, no. 2, pp. 977-994, 2017.

[11] X. He, H. Geng, and G. Yang, "Equivalent modelling of wind farm for small-signal stability analysis in weak power system," Journal of Engineering, vol. 2017, no. 13, pp. 1399-1393, 2017.

[12] Ö. Göksu, R. Teodorescu, C. L. Bak, F. Iov, and P. C. Kjær, "Instability of wind turbine converters during current injection to low voltage grid faults and PLL frequency based stability solution," IEEE Trans. Power Syst., vol. 29, no. 4, pp. 1683-1691, 2014.

[13] D. Dong, B. Wen, D. Boroyevich, and P. Mattavelli, "Analysis of phase-locked loop low-frequency stability in three-phase gridconnected power converters considering impedance interactions," IEEE Trans. Ind. Electron., vol. 62, no. 1, pp. 310-321, 2015.

[14] H. Yuan, H. Xin, K. Wang, Z. Wang, L. Xu, and H. Xie, "Instability mechanism analysis of inverters connected to weak grid during severe voltage sag on remote grid side," Automation of Electric Power Systems, vol. 42, no. 22, pp. 38-43, 2018.

[15] H. Yuan, H. Xin, L. Huang, Z. Wang, and D. Wu, "Stability analysis and enhancement of type- 4 wind turbines connected to very weak grids under severe voltage sags," IEEE Trans. Energy Convers., in press.

[16] Q. Hu, J. Hu, H. Yuan, and H. Tang, "Synchronizing stability of DFIG-based wind turbines attached to weak AC grid," in Proc. ICEMS, 2014, pp. 2618-2624. 
[17] C. Zhang, X. Cai and Z. Li, "Transient stability analysis of wind turbines with full-scale voltage source converter," CSEE J. Power Energy Syst., vol. 37, no. 14, pp. 4018-4026, Jul. 2017.

[18] G. Han, C. Zhang, and X. Cai, "Mechanism of frequency instability of full-scale wind turbines caused by grid short circuit fault and its control method," Trans. China Electrotechnical Society, vol. 33, no. 10, pp. 2167-2175, May 2018.

[19] H. Wu and X. Wang, "Design-oriented transient stability analysis of grid-connected converters with power synchronization control," IEEE Trans. Ind. Electron., in press.

[20] H. Wu and X. Wang, "Transient angle stability analysis of gridconnected converters with the first-order active power loop," in Proc. 2018 IEEE Appl. Power Electron. Conf. Expo. (APEC), Mar. 2018.

[21] H. Wu and X. Wang, "Transient stability impact of the phase-locked loop on grid-connected voltage source converters," in Proc. 2018 Int. Power Electron. Conf. (IPEC-Niigata 2018-ECCE Asia), May 2018.

[22] H. Wu and X. Wang, "An adaptive phase-locked loop for the transient stability enhancement of grid-connected voltage source converters," in Proc. 2018 IEEE Energy Convers. Congr. Expo. (ECCE), Sept. 2018.

[23] M. Kabalan, P. Singh, and D. Niebur, "Nonlinear Lyapunov stability analysis of seven models of a DC/AC droop controlled inverter connected to an infinite bus," IEEE Trans. Smart Grid, vol. 10, no. 1, pp. 772-781, Jan. 2019.

[24] Z. Shuai, C. Shen, X. Liu, Z. Li, Z. J. Shen, "Transient angle stability of virtual synchronous generators using Lyapunov's direct method," IEEE Trans. Smart Grid, in press.

[25] B. Shakerighadi, E. Ebrahimzadeh, F. Blaabjerg, and C. Leth Bak, "Large-signal stability modeling for the grid-connected VSC based on the Lyapunov method," Energies, vol. 11, no. 10, pp. 2533, Sept. 2018.

[26] M. Kabalan, P. Singh, and D. Niebur, "Large signal Lyapunov-based stability studies in microgrids: A review," IEEE Trans. Smart Grid, vol. 8, no. 5, pp. 2287-2295, Sept. 2017.

[27] X. He, H. Geng, and G. Yang, "Synchronization stability analysis of grid-tied power converters under severe grid voltage sags," in Proc. IEEE PEAC, 2018

[28] M. G. Taul, X. Wang, P. Davari, and F. Blaabjerg, "Grid synchronization of wind turbines during severe symmetrical faults with phase jumps," in Proc. 2018 IEEE Energy Convers. Congr. Expo. (ECCE), Sept. 2018.

[29] S. Golestan, J. M. Guerrero, and J. C. Vasquez, "Three-phase PLLs: A review of recent advances," IEEE Trans. Power Electron., vol. 32, no. 3, pp. 1894-1907, Mar. 2017.

[30] X. He, H. Geng, G. Yang, "A generalized design framework of notch filter based frequency-locked loop for three-phase grid voltage," IEEE Trans. Ind. Electron., vol. 65, no. 9, pp. 7072-7084, 2018.

[31] X. He, H. Geng, and G. Yang, "Reinvestigation of single-phase FLLs," IEEE Access, vol. 7, pp. 13178-13188, 2019.

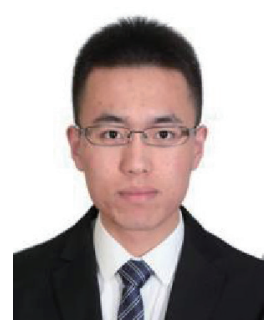

Xiuqiang He received the B.S. degree in automatic control from the Department of Automation, Tsinghua University, Beijing, China, in 2016. He is currently working toward the Ph.D. degree in control science and engineering at the Department of Automation, Tsinghua University.

His current research interests include gridsynchronization stability as well as transient stability of power electronic converters, and dynamic equivalent modeling of large-scale distributed generation systems.

Mr. He was a recipient of the Chinese National Scholarship, and the IEEE International Power Electronics and Application Conference and Exposition (PEAC) Excellent Paper Award in 2018

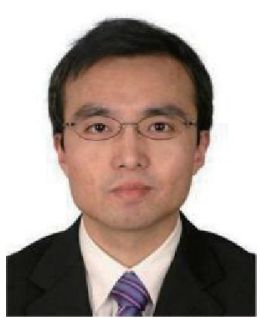

Hua Geng received the B.S. degree in electrical engineering from Huazhong University of Science and Technology, Wuhan, China, in 2003, and the $\mathrm{Ph} . \mathrm{D}$. degree in control theory and application from Tsinghua University, Beijing, China, in 2008.

From 2008 to 2010, he was a Postdoctoral Research Fellow with the Department of Electrical and Computer Engineering, Ryerson University, Toronto, ON, Canada. He joined the Department of Automation, Tsinghua University, in June 2010, where he is currently an Associate Professor. His current research interests include advanced control of power electronics and renewable energy conversion systems.

Dr. Geng serves as an editor of IEEE Transactions on Energy Conversion, an associate editor of IEEE Transactions on Industry Applications and an associate editor of Chinese Journal of Electrical Engineering.

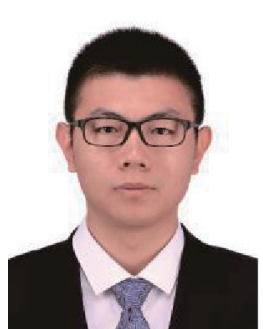

Shaokang Ma received the B.S. degree from the College of Electrical and Information Engineering, Hunan University, Changsha, China, in 2012, and the M.S. degree in 2015 from the Department of Automation, Tsinghua University, Beijing, China, where he has been working toward the Ph.D. degree since 2015 .

His current research interests include distribution generation systems, renewable energy conversion systems, and digital control systems. 